pag

Business School

WORKING PAPER SERIES

Working Paper

2014-246
Comparaison des formations à la diversité : la perception des étudiants et des professionnels est-elle un aiguillon vers l'action?

Marie José Scotto

Linda Prince

Ridha Chakroun

André Boyer

http://www.ipag.fr/fr/accueil/la-recherche/publications-WP.html

IPAG Business School

184, Boulevard Saint-Germain

75006 Paris

France 


\title{
Comparaison des formations à la diversité : la perception des étudiants et des professionnels est-elle un aiguillon vers l'action?
}

\author{
Marie José Scotto ${ }^{a}$, Linda Prince ${ }^{a}$, \\ Ridha Chakroun ${ }^{\mathrm{ab}}$, André Boyer ${ }^{\mathrm{ab}}$ \\ ${ }^{\mathrm{a}}$ IPAG Business School \\ ${ }^{\mathrm{b}}$ Université de Nice
}

\section{Résumé}

La prise en compte de la diversité devient une compétence sociale importante en entreprise. La formation à la diversité reste hétérogène et peu évaluée en France. Nous avons dans un premier temps établi un état des lieux de l'offre de formation en France et en Grande Bretagne puis nous avons évalué la perception de la formation à la diversité au sein d'un échantillon d'étudiants internationaux. Dans un deuxième temps, nous avons apprécié dans une étude exploratoire les concordances et discordances qu'étudiants et professionnels vivant en France pouvaient avoir vis-à-vis de leur formation. Les taux de formation des étudiants français sont proches de ceux déclarés par les étudiants internationaux : $46 \%$ vs $40 \%$ pour la formation à la RSE et $54 \%$ vs $42 \%$ pour la formation à la diversité. Toutefois, avec $14 \%$ des effectifs des professionnels se déclarant formés ou informés sur la diversité, il apparaît qu'en attendant le renforcement des pratiques de diversité en entreprise par l'arrivée d'étudiants spécifiquement formés, une implication des professionnels conforme aux cadres théoriques mobilisés passe par un accroissement de la formation continue afin de lutter contre la rémanence des stéréotypes et la réalité des situations de discrimination. Une telle approche permet de passer la diversité en entreprise des pratiques déclaratives à des pratiques intégrées.

\begin{abstract}
The awareness of the need for diversity is becoming an important social skill for companies. Training is this area appears mixed and undervalued in France. The first phase of the research sought to establish the availability of training courses in France and in Great Britain (to provide a comparison) coupled with a further study evaluating the perception of diversity management training on a group of international students. This research was followed up by an exploratory study of the differences in opinion held by students and professionals in France of their training in this area. The extent of the training received by French students was found to be similar to that of the international students, where training in Corporate Social Responsibility was found to be $46 \%$ in France and $40 \%$ for the international students and 54\% in France versus 42\% for the international group concerning Diversity Management. However, with only 14\% of the professionals studied claiming to have received information or training in diversity management, it appears that until the arrival of new graduates trained in this area to reinforce the practice of diversity management in the company, professionals conforming to existing theoretical frameworks will contribute to the growth in executive education in order to counter the retention of stereotypes and discriminatory behaviour. Such an approach allows company diversity to pass from being a positive statement to an integrated practice.
\end{abstract}

Post Print : Management \& Avenir, n²8, septembre-octobre 2009. 
Les théories managériales de la diversité s’imposent fortement en France. Plus de 1700 entreprises ont signé en France, la Charte de la Diversité au mois d'avril 2008. Le management de la diversité devient pour les gestionnaires de Ressources Humaines une compétence nouvelle à maîtriser. Pour autant, les formations managériales françaises ont elles intégrés ces enseignements à leur cursus de formation, notamment pour les futurs gestionnaires de ressources humaines? Notre première partie rappellera les éléments importants des théories de la diversité et insistera sur le rôle de la fonction des ressources humaines dans l'intégration de la diversité au sein de la force de travail. Dans une seconde partie, nous détaillerons l'offre des enseignements en France et en Grande Bretagne concernant le management de la diversité. Le choix de la Grande Bretagne se justifie par le fait que le concept de diversité possède une plus grande antériorité dans les pays anglosaxons. Enfin, nous présenterons une étude exploratoire sur trois échantillons: des étudiants internationaux en semestre d'échange en Ecole de Commerce, des étudiants et des professionnels exerçant en France.

\section{Cadre théorique de la diversité}

\subsection{L’approche managériale de la diversité}

Le concept de diversité a été développé par les chercheurs anglo-saxons, à partir d'une analogie avec les sciences du vivant et le concept de biodiversité. ${ }^{1}$ Les auteurs ont identifiés les différentes dimensions de la diversité : du triple axe de Rijamampianina et Carmichael (2005) aux six dimensions primaires de Litvin (1997). Les principales dimensions de la diversité, telles que les auteurs les présentent, se structurent entre diversité visible (sexe, âge, ou origine) et diversité invisible liés à des éléments de formation, de compétences, d'origine sociale ou d'orientation sexuelle (Millikens et Martins, 1996). Ces dimensions peuvent tout à fait se combiner, ce qui rend cette notion de diversité particulièrement complexe. Selon Semache (2006), l'émergence de ce concept de diversité s'articule avec une évolution au sein de l'organisation du groupe vers l'individu. Il reviendra à Thomas et Ely (1996) d'avoir présenté la diversité comme une approche des différences et des similitudes de la force de travail, au sein de l'organisation mais également de l'avoir intégré à une logique d'apprentissage de l'organisation. L’hétérogénéité du capital humain de l'entreprise devient ainsi un atout que l'entreprise se doit de valoriser et de maximiser. Ce modèle "libéral » s'appuie à notre sens sur l'approche « ressource based » de l'entreprise qui s'est développée aux Etats -Unis dans les années 1990, ce qui à conduit à développer la logique du business case de la diversité (Frinck et alii, 2003). Ici, le point focal est constitué par la ressource humaine et organisationnelle que Grant (1991) et Barney (1991) ont fait figurer parmi les ressources les plus stratégiques. C'est dans la variété démographique de la force de travail que l'entreprise pourra développer une ressource nouvelle, compétitive, et spécifique, dont ne disposera pas une organisation concurrente qui privilégie une structure démographique plus homogène (Frinck et alii, 2004).

\subsection{Le rôle central de la Fonction Gestion des Ressources Humaines}

La fonction RH se trouve donc au cœur de ce management de la diversité (Konrad et Linnehan, 1995) et doit donc piloter un véritable processus de mise en œuvre de la diversité dans l'entreprise en articulant ses différentes dimensions. Ce processus nécessite de mobiliser

\footnotetext{
${ }^{1}$ La biodiversité s’entend comme la totalité de toutes les variations des êtres vivants. Elle se définit également comme la dynamique des interactions dans des milieux en changement.
} 
des ressources humaines et financières. Nous pouvons reprendre le schéma de Gisbert (2007) qui présente les différentes étapes d’une démarche globale de diversité.

\section{Figure 1 : processus de mise en œuvre de la démarche d'égalité professionnelle}

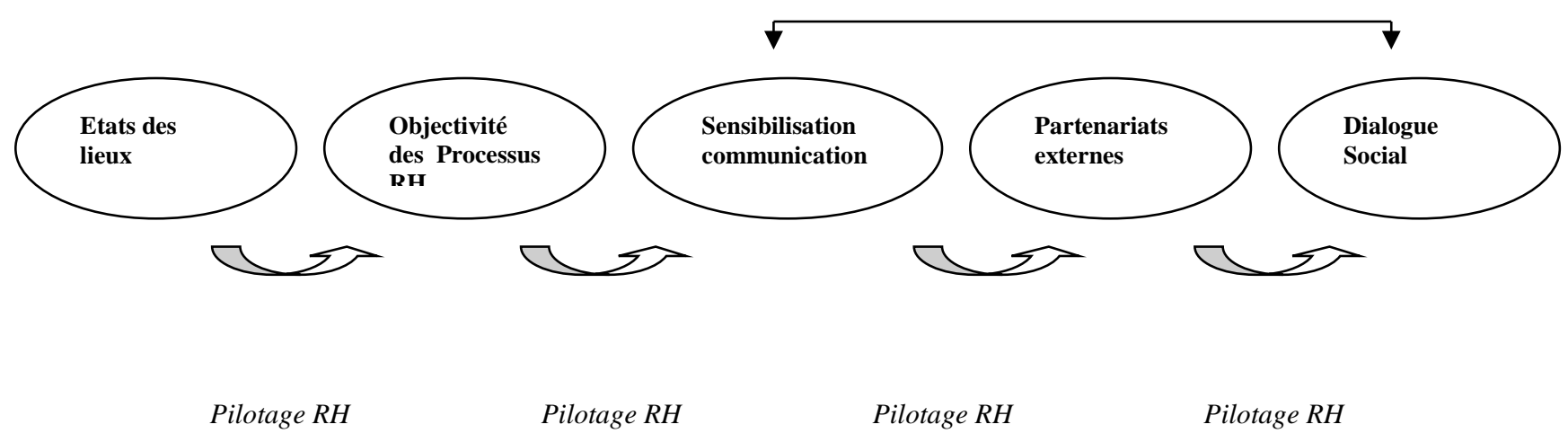

Source: d'après Gisbert, 2007. EMCC Company Network Seminar.

La démarche de diversité suggère donc une refonte de tous les processus $\mathrm{RH}$ pour les rendre « diversity conscious ». En filigrane, nous voyons apparaître l'évolution de la fonction RH. Ainsi que le souligne Picq (2005), la fonction RH à l'instar de la fonction marketing évolue de la GRH «de masse », vers une gestion de groupes différenciés (femmes, employés issus des minorités ethniques, seniors ...), vers une logique d'individualisation de plus en plus poussée, que Liff (1997) qualifie de GRH de « cibles ». La fonction RH dans cette logique se trouve confrontée à trois défis : la valorisation de la diversité individuelle dans un cadrage d'innovation et de changement, le développement d'équipes « diversifiées » afin d'augmenter la capacité collective de la force de travail et enfin l'adaptation des modes opératoires des ressources humaines à des structures d'organisation, elles mêmes en mouvement (Picq, 2005). Finalement, ce sont l'ensemble des pratiques RH qui se trouvent concernées : le recrutement, l'évolution professionnelle, la formation, la rémunération et l'articulation entre la vie familiale et la vie professionnelle. Ce dernier élément concerne plus spécifiquement la dimension "genre » de la diversité, dont nous pensons qu'elle implique des spécificités au sein de l'ensemble de la diversité. Le schéma ci-dessus montre bien que la phase de diagnostic est un passage obligé pour permettre d'identifier les raisons explicites ou implicites de sous-représentation de certains groupes. Cette phase de diagnostic n'est pas aussi évidente qu'il y parait. Tout d'abord, il importe de bien définir l'adéquation des données à recueillir avec les différentes dimensions de la diversité que l’on souhaite développer. En clair, de quelle diversité parle- t- on ? Des problèmes autres que la collecte et le traitement des informations peuvent également se poser. On le constate à propos des réactions récentes concernant Air France et l'utilisation des statistiques ethniques. La ou l'entreprise se réclame d'une action au nom de l'équité et de la diversité, d'autres ${ }^{2}$ y voit des critères « illégitimes et illégaux », et rappellent la décision du Conseil Constitutionnel du 15 novembre 2007, invalidant l'article sur les statistiques ethniques contenu dans la loi sur l'immigration. En France, donc, il semble que les statistiques faisant état de l'origine ethnique des salariés soient considérés comme particulièrement sensibles, voire suspectes, même si la $\mathrm{CNIL}^{3}$ se positionne comme favorable à une évolution sur ce point, et que l'entreprise se défende en invoquant le caractère non obligatoire et soumis à l'accord du salarié de ce type de données

\footnotetext{
${ }^{2}$ Syndicats CFDT et Sud, association SOS Racisme

${ }^{3}$ Commission Nationale Informatique et Libertés
} 
(AFP, 2007). Les politiques de recrutement constituent un des relais de la politique de diversité dans l'entreprise, avec tout d'abord des viviers de recrutement élargis et la prise de conscience des risques de discrimination existant. Leur diminution passe par la formation des recruteurs et des définitions précises de critères, le CV anonyme n'étant qu'un outil utile dans le cadre de l'objectivité des processus certes, mais limité au premier tri des candidatures. Associé à ces pratiques de recrutement, la démarche de diversité au sein d'une entreprise doit s'appuyer sur la mise en perspective des modes d'évaluation et d'évolution de carrière. La formation et l'évaluation des managers sont essentielles (Kandolla et Fullerton, 2004) à cet égard afin de permettre la réévaluation systématique et sans complaisance, des critères d'appréciation des compétences (Ghuilamila et Levet, 2006). Certains auteurs évoquent la possibilité d'une "médiation " nécessaire d'un professionnel $\mathrm{RH}$ permettant de ne plus réduire l'évaluation du salarié à une relation de face à face hiérarchique (Ghuilamila et Levet, 2006). Un troisième axe présenté dans la démarche consiste à structurer une politique de formation et de communication envers les partenaires externes, les partenaires sociaux et les salariés pour sensibiliser les uns et les autres à la permanence des stéréotypes, à la réalité des situations de discrimination, mais également aux possibilités de prise en compte sans a priori, de mesures de discrimination positive. Et ceci n'est pas la chose la plus aisée. Ainsi, nous constatons au travers de ce panorama succinct, la complexité que la mise en place d'une démarche de diversité implique, si elle veut dépasser le stade de l'intention et du discours et atteindre la «culture d'inclusion » dont parle Thornburg (1994), en référence au paradigme " learning and effectiveness » de Thomas et Ely (1996). Cependant, au vu de la "nouveauté » relative de ces théories et de leurs traductions concrètes sur le terrain de l'entreprise, ne peuton pas supposer que les formations en gestion et notamment les formations en gestion des ressources humaines puissent rester plus classiquement sur des schémas globaux de la «GRH de masse », au sens de Liff (1997)? Les futurs gestionnaires des ressources humaines sont ils préalablement formés à appréhender ces approches complexes et contradictoires ?

\section{L’enseignement du management de la diversité : une comparaison}

\section{France - Grande Bretagne.}

Cette enquête s'est appuyée sur une recherche documentaire menée sur deux sites britanniques et un site français. Pour la Grande Bretagne, nous avons utilisé les informations du site : www.ucas.ac.uk, qui répertorie plus de 300 programmes nationaux universitaires au niveau licence (Bachelor). En ce qui concerne les programmes masters de ces mêmes universités, ils sont répertoriés sur le site www.prospects.ac.uk. En France, nous avons constaté que les sources d'information sont plus parcellaires et plus difficiles à identifier. Nous avons néanmoins trouvé que le site www.campusfrance.org permettait d'obtenir des informations globales sur les formations proposées par les différentes institutions (Universités et Ecoles de Commerce). Ce site se rapproche le plus, dans sa conception, des deux sites britanniques. Le moteur de recherche est cependant différent. Les sites britanniques permettent une recherche plus pointue.

\subsection{Un choix restreint de l'offre de cours en Grande - Bretagne}

La recherche a permis de montrer que les formations licence n'offrent pas de programmes spécifiques orientée sur la gestion de la diversité. Néanmoins, il convient de moduler cette remarque. Les licences spécialisées en Management des Ressources Humaines proposent des 
modules complets dédiés au thème de la gestion de la diversité. Les autres licences de gestion (Finance, marketing, logistique, droit) incluent un module de gestion des ressources humaines qui abordent le thème de la diversité, afin de sensibiliser et d'ouvrir les étudiants à ces concepts. Nous avons plus particulièrement étudié l'offre masters. Nous présentons dans le tableau ci-dessous, les formations spécialisées qui existent dans l'offre d'enseignement national en Grande-Bretagne. Sur plus de 300 universités, nous avons répertorié deux universités intégrant ces programmes dans leur enseignement de gestion. Ces deux masters sont proposés en gestion, par les Universités Queen Mary College London et Leeds University Business School. L'université de London Metropolitan, quant à elle, intègre ces formations spécifiques à la diversité, dans sa faculté de sciences sociales. Il est important de noter que l'Université de Queen Mary College London, héberge un centre national d'études sur l'égalité et la diversité. Ce centre travaille sur ce thème depuis plus de 6ans, si l'on se réfère à leur liste de publications, ce qui explique l'importance accordée à la diversité dans les programmes de cette université. Cinq autres universités en Grande Bretagne proposent des modules électifs spécialisés dans le management de la diversité au sein de certaines formations en management, notamment les Universités de Leeds et de Glasgow.

\subsection{L’offre de cours au niveau licence et masters en France : pas encore intégrée ?}

Les informations ont été recueillies au travers du site campusfrance.org, qui répertorie les formations supérieures en France, en gestion et management. Nous avons constaté, qu'en ce qui concerne les niveaux de formation en Ressources Humaines, en Licence, Master1, Master 2 et Master spécialisé, aucune formation spécifique à la diversité/égalité professionnelle, n’est répertoriée. Nous avons poursuivi notre recherche au niveau des syllabus des différentes formations en ressources humaines à tous les niveaux. Nous n'avons identifié aucun électif ou sous module spécifiquement présenté comme dédié à ce thème de la diversité et/ou de l'égalité professionnelle. Les principaux électifs proposés restent très techniques et concernent principalement des modules tels que gestion de paye. Même les modules traitant des techniques de recrutement ne font pas référence explicite au management de la diversité globale. La seule formation répertoriée, traitant des diversités culturelles est un Master professionnel en Sciences Humaines et Sociales à l'Université de Clermont-Ferrand II. Il est tout à fait possible que des formations en gestion des ressources humaines, traitant du thème spécifique de la diversité, existent dans d'autres établissements, mais elles ne sont pas répertoriées dans la liste des formations supérieures proposée par le site campusfrance.org. au cours de l'année 2008. En Grande Bretagne, les formations à la diversité émergent d'un champ de recherche robuste et ancien, celui des gender studies, qui ont essaimé vers les formations de gestion à partir des sciences sociales, en parallèle avec le développement d'un cadre législatif favorable à la diversité et à l'égalité des chances. En France, la thématique apparaît encore récente dans les recherches de gestion, ce qui peut expliquer que l'offre d'enseignement soit si difficile à identifier, selon les données auxquelles nous avons eu accès. Pour autant, cela n’implique pas que les étudiants français ne soient pas sensibilisés ou n’aient pas eu de formation sur ce thème de la diversité et plus globalement de la responsabilité sociale d'entreprise. Simplement, à la différence de la Grande Bretagne, ce thème n’apparaît pas dans les formations RH de manière explicite, mais a pu être traité en thème transversal dans l'étude des différentes fonctions des ressources humaines. Cette hypothèse semble être confirmée par les résultats de notre étude sur l'échantillon d'étudiants français où $54 \%$ des étudiants interrogés disent avoir bénéficié d'au moins une information ou formation sur la diversité. 
Tableau 1 : les formations masters spécialisées en Grande-Bretagne

\begin{tabular}{|c|c|c|c|c|}
\hline Université & Diplôme & Titre & Faculté & $\begin{array}{l}\text { Electifs } \\
\text { (modules optionnels) }\end{array}$ \\
\hline \multicolumn{5}{|l|}{$\begin{array}{l}\text { Formations } \\
\text { spécifiques }\end{array}$} \\
\hline $\begin{array}{l}\text { Queen Mary, } \\
\text { London }\end{array}$ & M.Sc. & $\begin{array}{l}\text { Equality and Diversity } \\
\text { Management }\end{array}$ & $\begin{array}{ll}\text { Business and } \\
\text { Management }\end{array}$ & \\
\hline Leeds & M.A. & Diversity Management & $\begin{array}{l}\text { Business and } \\
\text { Management }\end{array}$ & \\
\hline $\begin{array}{l}\text { London } \\
\text { Metropolitan }\end{array}$ & M.A. & Equality and Diversity & Humanities & \\
\hline \multicolumn{5}{|l|}{$\begin{array}{l}\text { Formations } \\
\text { Généralistes }\end{array}$} \\
\hline Glasgow & M.Sc. & $\begin{array}{l}\text { Human } \quad \text { Resource } \\
\text { Management }\end{array}$ & Business & $\begin{array}{l}\text { Managing Diversity and } \\
\text { equal opportunities }\end{array}$ \\
\hline Glasgow & M.Sc. & $\begin{array}{l}\text { Management with } \\
\text { International Finance }\end{array}$ & Business & $\begin{array}{l}\text { Managing Diversity in } \\
\text { organisations }\end{array}$ \\
\hline York & M.A. & $\begin{array}{l}\text { Leadership and } \\
\text { Management }\end{array}$ & Management & Cultural Diversity \\
\hline $\begin{array}{l}\text { Kings College, } \\
\text { London }\end{array}$ & M.Sc. & $\begin{array}{l}\text { H.R.M. and } \\
\text { Organisational analysis }\end{array}$ & Management & $\begin{array}{l}\text { The New Workforce- } \\
\text { Managing Diversity }\end{array}$ \\
\hline Greenwich & M.A. & International H.R.M. & Business & Managing Diversity \\
\hline Leeds & M.A. & $\begin{array}{ll}\text { Human } & \text { Resource } \\
\text { Management } & \end{array}$ & Business & Gender and Equality at work \\
\hline Essex & M.Sc. & $\begin{array}{l}\text { Accounting, Finance and } \\
\text { Management }\end{array}$ & Business & $\begin{array}{l}\text { Significance of Gender as } \\
\text { organising principles }\end{array}$ \\
\hline
\end{tabular}

Source : adapté d’après données récoltés sur site www.prospects.ac.uk, 2008.

\section{Perception du concept de diversité : une première enquête exploratoire}

\subsection{Contexte, objectif et méthodologie}

Cette première enquête exploratoire a été menée auprès d'une population d'étudiants internationaux participants à l'IBMP (International Business and Management Programme) ${ }^{4}$ de l'Ipag, des étudiants français en gestion de l'Université de Nice. L'objectif de cette étude a été d'identifier le niveau de compréhension des étudiants concernant les concepts de responsabilité sociale d'entreprise et de diversité. Nous souhaitions également connaître s’ils avaient suivi des enseignements spécifiques ou des modules intégrés dans d'autres cours, et

\footnotetext{
${ }^{4}$ Ce programme accueille les étudiants en échange des universités partenaires de l’Ipag.
} 
quelle importance était attribuée par les étudiants aux différentes dimensions de la diversité dans les politiques de recrutement et d'évolution professionnelle. L'étude cherchait également à mesurer le degré de connaissance des étudiants concernant les différentes obligations légales de la mise en œuvre de la diversité dans les entreprises : quotas éventuels, politiques de recrutement ciblées... Nous avons également demandé aux étudiants de préciser, quelle était, la structure d'un CV type dans leur pays d'origine, indépendamment de l'expérience professionnelle et des compétences. Les questionnaires ont été administrés au premier semestre 2008 lors d'une séance du cours de « Small Business Development » à l'Ipag à Nice, auprès d'une population de 45 étudiants qui viennent de terminer deux années d'études universitaires en gestion.

\subsection{Les résultats}

Les étudiants internationaux venaient de 8 pays européens (Irlande, Grande Bretagne, Allemagne, Espagne, Pologne, France, Pays Bas, Suisse), du Mexique, du Canada, des Etats Unis et de Corée. On peut noter une répartition équilibrée de la provenance des différents étudiants. Le groupe ne comportait aucun étudiant handicapé ni étudiant senior.

Tableau 2 : description de l'échantillon des étudiants internationaux

\begin{tabular}{|l|l|l|l|l|l|}
\hline $\begin{array}{l}\text { Nombre } \\
\text { d'étudiants }\end{array}$ & Provenance & $\begin{array}{l}\text { Population } \\
\text { masculine }\end{array}$ & $\begin{array}{l}\text { Population } \\
\text { féminine }\end{array}$ & Age & Handicap \\
\hline 45 & 12 pays & $30 \%$ & $70 \%$ & $\begin{array}{l}20 \text { à } 24 \\
\text { ans }\end{array}$ & 0 \\
\hline
\end{tabular}

Afin de ne pas dénaturer le sens intime des réponses apportées par les étudiants internationaux à notre questionnaire posé en anglais, nous proposons une analyse des réponses en maintenant des items en anglais.

Question 1 :

What is Corporate Social Responsibility?

Dont Know

$18 \%$ of sample

( $28 \%$ for males, $13 \%$ for females)

Response groupings:

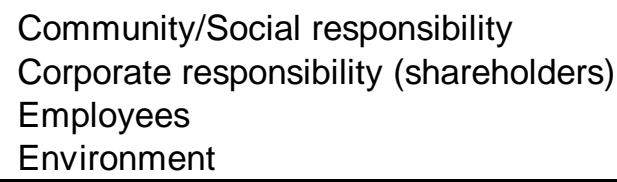

\author{
$38 \%$ of sample \\ $20 \%$ of sample \\ $16 \%$ of sample \\ $8 \%$ of sample
}

Parmi les 18\% d'étudiants qui n’ont pas d'idées sur le sujet, on note que les garçons sont les plus nombreux. La majorité a répondu positivement. Ces réponses ont été triées en quatre catégories. La partie la plus importante de l'échantillon associe le concept de CSR ( Corporate Social Responsibility) que nous traduirions en français par Responsabilité Sociale d'Entreprise (RSE), avec une responsabilité sociale au sein de la société civile et pas forcément en liaison avec l'entreprise. $1 / 5$ des étudiants interrogés, associe ce concept à la responsabilité de l'entreprise envers ses actionnaires. Seulement $16 \%$ de l'échantillon 
considère que la notion de CSR est liée à des obligations de l'entreprise envers ses salariés. Un petit groupe a cité l'environnement comme étant de la responsabilité de l'entreprise. La distribution des réponses n'a pas fait apparaître de corrélations avec la provenance des étudiants ou le genre.

Question 2:

What is Diversity in the Workplace?

Dont know

$4 \%$ of sample

Response groupings:

Nationalities and cultures

Race, religion, gender, age

Understanding other people

Variety of tasks at work
$45 \%$ of sample

$26 \%$ of sample

$15 \%$ of sample

$10 \%$ of sample

Parmi les 4\% d'étudiants qui n’ont pas d'idées sur le sujet, on note à nouveau que les garçons Les plus nombreux, néanmoins la notion de diversité parle beaucoup plus aux étudiants qui ont répondu positivement à $96 \%$. Ces réponses ont été triées en quatre catégories qui représentent aux yeux des étudiants les dimensions de la de la diversité. Quasiment la moitié de l'échantillon associe diversité avec nationalités et cultures. Si l'on intègre les $15 \%$ ayant répondu sur la compréhension mutuelle, on constate que pour les étudiants la dimension principale de la diversité porte sur l'inter-culturalité. Il est à noter que ces réponses sont essentiellement données par les étudiants masculins. Les principales dimensions visibles de la diversité : race, genre et âge ont été citées par $26 \%$ de notre échantillon ainsi que la dimension religion. Les étudiants qui associent diversité et multiplicité des tâches et des lieux de travail sont des filles. La distribution des réponses n’a pas fait apparaître de relations en fonction de la provenance des étudiants.

Question 3

Has Diversity been mentioned in your courses?

Number of students

Yes 35

No 6

Dont know 4

Question 4

in which courses?

\begin{tabular}{lc} 
& Numbers of calls \\
Human Resources & 26 \\
Marketing & 18 \\
Law & 7 \\
Intercultural Management & 9 \\
Finance & 2 \\
Ethics & 2 \\
Business communication & 1 \\
Organisational Behaviour & 1 \\
Business Strategy & 1 \\
Dont know & 1 \\
\hline
\end{tabular}

Les réponses montrent que les étudiants de l'échantillon ont majoritairement eu une sensibilisation à la notion de diversité dans leurs enseignements, même si le concept n'est pas complètement intégré. Les réponses ne montrent aucune corrélation avec le genre ou la 
nationalité. Majoritairement, lorsque la notion de diversité a été vue, elle l'a été durant les cours de gestion des ressources humaines et de marketing. Les citations concernant les cours de droit proviennent essentiellement des étudiants néerlandais et canadiens.

\begin{tabular}{ll} 
Question 5 & Is there a C.S.R. module on your course? \\
\hline Yes & \% of total students \\
No & $40 \%$ \\
Dont know & $20 \%$ \\
& \multicolumn{2}{c}{ Is there a D.I.W. module on your course? } \\
\multicolumn{3}{|l}{ \% of tolal students } \\
Yes & $42 \%$ \\
No & $38 \%$ \\
Dont know & $20 \%$ \\
\hline
\end{tabular}

En ce qui concerne la formation à la "Corporate Social Responsability », les réponses sont équitablement réparties entre les oui et les non. Une des explications que nous pourrions avancer serait que les étudiants de l'échantillon sont en troisième année et n’ont pas pu encore avoir les cours spécialisés. A noter cependant, que tous les étudiants canadiens et suisses ont répondu positivement pour le cours de "Corporate Social Responsibility ». La seconde partie de la question concernait la diversité sur le lieu de travail. La majorité y est sensibilisée. Néanmoins, nous avons remarqué des différences notables selon les nationalités. Les Espagnols ont globalement répondu non à cette partie de la question. Tous les canadiens et les suisses ont répondu oui, ce qui parait cohérent avec leurs réponses précédentes. La plupart des réponses « ne sait pas » provient des étudiants britanniques.

Questions 6 \& 7:

Rate importance of elements: average value.

\begin{tabular}{|c|c|c|c|c|}
\hline & \multicolumn{4}{|c|}{ ( out of $\max =7$ ) } \\
\hline & \multicolumn{2}{|c|}{ Recruitement policy } & \multicolumn{2}{|c|}{ Career development } \\
\hline & Females & Males & Females & Males \\
\hline Gender & 6 & 6 & 5,3 & 5,4 \\
\hline Handicap & 4,9 & 5,7 & 5 & 4,7 \\
\hline Age & 4,8 & 5,6 & 4,8 & 4,5 \\
\hline Race & 5,2 & 5,5 & 5,8 & 5,2 \\
\hline Sex orientation & 3,6 & 3,9 & 4 & 2,8 \\
\hline
\end{tabular}

En matière de politique de recrutement, nous notons que les résultats moyens masquent chez les femmes une plus grande dispersion des scores d'importance que chez les hommes. En matière de diversité face aux recrutements, les scores d'importance extrêmes sont identiques quelque soit le genre. Le genre apparaît ainsi comme l'item le plus important et l'orientation sexuelle comme l'item le moins important. Nous avançons l'hypothèse que l'orientation sexuelle faisant partie des éléments de diversité «invisible » au sens de Millikens et Martins (1996), elle serait moins perçue par les étudiants. En matière de politique de développement de carrière les scores d'importance féminins sont généralement plus élevés que les scores masculins sur l'ensemble des items. Les femmes ont notamment attribué des scores moyens supérieurs à 4 et plus homogènes que les hommes pour l'ensemble des items de diversité évalués dans les procédures de développement de carrière. Enfin, il résulte de l'analyse des deux contextes précédents que l'amplitude des scores d'importance pour les procédures de recrutement et de développement de carrière est plus large chez les hommes que chez les 
femmes comme le montre la figure 2. Les étudiants semblant ainsi plus sensibilisés à la prise en compte de la diversité lors des recrutements alors que les étudiantes y seraient plus attentives lors des situations de recrutement comme d'évolution de carrière. Ce phénomène est particulièrement accentué dans le contingent espagnol des étudiants et pourrait être expliqué par l'évolution récente des mentalités en Espagne avec le développement des processus d'égalité professionnelle, de parité et de diversité dans ce pays.

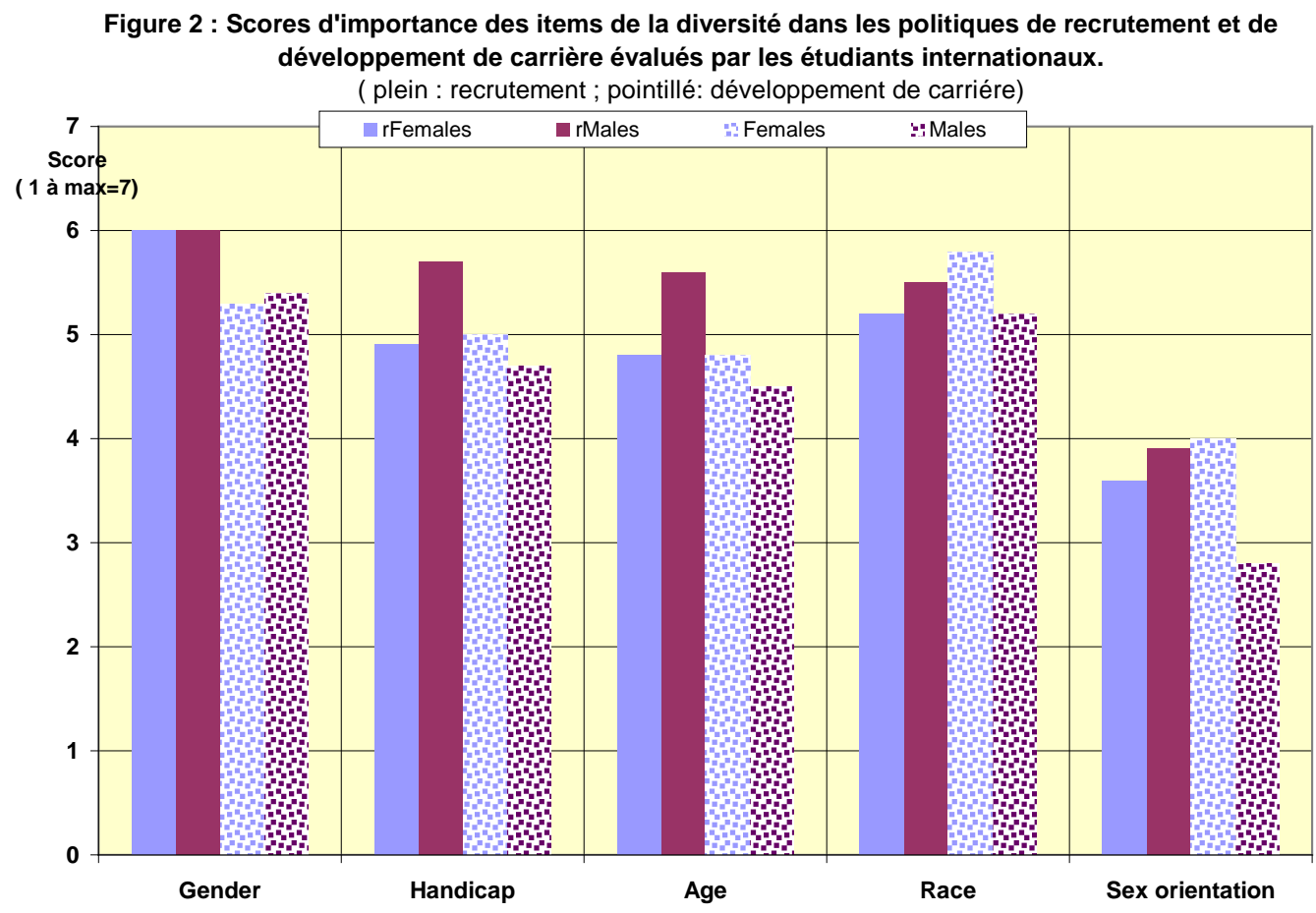

\begin{tabular}{lc} 
Question 8 & $\begin{array}{c}\text { Are there laws in your country concerning } \\
\text { DVIERSITY in the workplace? }\end{array}$ \\
\hline Yes & $\% 4 \%$ \\
No & $6 \%$ \\
Dont know & $30 \%$ \\
\hline
\end{tabular}

Les étudiants de l'échantillon sont sensibilisés à la question de la diversité sur le lieu de travail. Les étudiants canadiens et américains ont dans leur totalité répondu positivement à cette question. Le fort pourcentage de non réponse («don't know») peut s'expliquer par le fait qu'une partie des étudiants de l'échantillon étudient dans un pays qui n’est pas leur pays d'origine, et donc ne disposent pas de l'information. 


\begin{tabular}{lcc} 
Question 9 & $\begin{array}{c}\text { Are companies legally obliged to apply diversity } \\
\text { quotas in their recruitment policies? }\end{array}$ \\
\hline Yes & \multicolumn{2}{c}{$\%$ of total students } \\
No & $46 \%$ \\
Dont know & $7 \%$ \\
\hline
\end{tabular}

On constate qu'un pourcentage élevé d'étudiants (près de la moitié de l'échantillon) ne connaît pas les obligations légales des entreprises de leur pays en matière de diversité lors des procédures de recrutement. Par contre, tous les étudiants suisses, américains et coréens ont répondu positivement à l'utilisation des quotas dans les entreprises de leurs pays. Ces modalités de réponses sont encore plus marquées quant les étudiants sont interrogés sur les obligations de diversité appliquées par les entreprises pour leur top management.

\begin{tabular}{ll} 
Question 10 & $\begin{array}{c}\text { Are companies legally obliged to apply diversity } \\
\text { quotas in their top management positions? }\end{array}$ \\
\hline Yes & $\begin{array}{l}\% \text { of total students } \\
\text { No }\end{array}$ \\
Dont know & $27 \%$ \\
\hline
\end{tabular}

Nous remarquons que la moitié des étudiants n’est pas informée. Quant à l'autre moitié, elle se répartit de manière équilibrée. Les réponses en fonction des nationalités sont variées entre les oui et les non. A cette question également, tous les étudiants ont répondu positivement.

On a C.V. in your country, do you include the

Question 11 following?

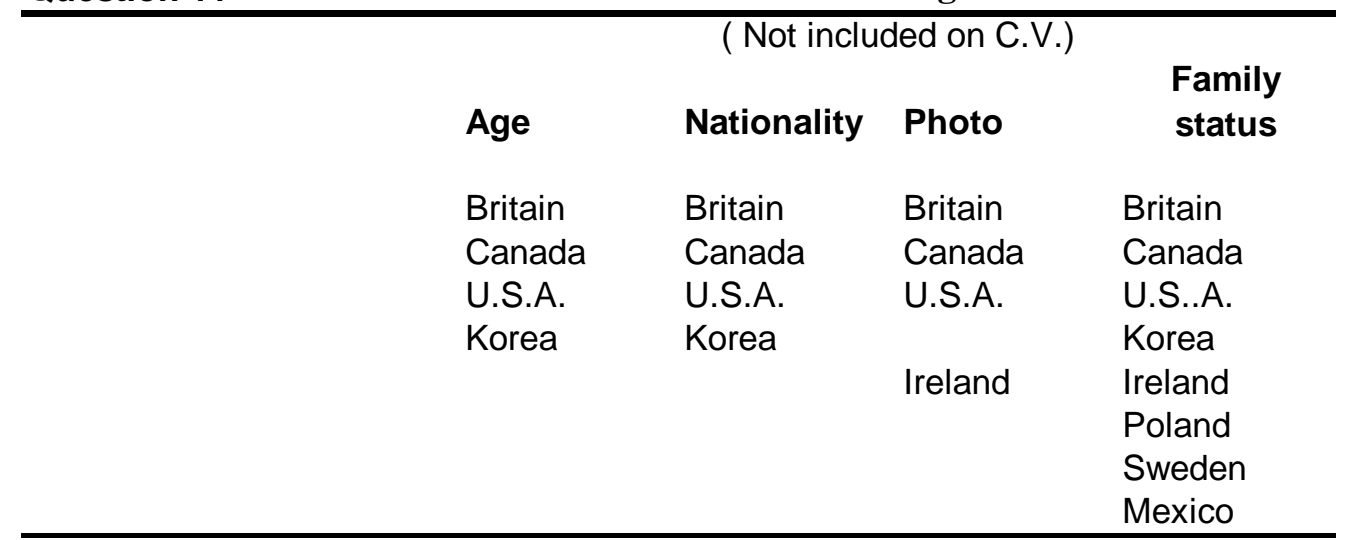

Par ailleurs les étudiants issus de Grande Bretagne du Canada et des USA ne mentionnent sur les CV, ni l'âge, ni la nationalité ni le statut familial. Les données personnelles n'apparaissent pas sur les CV classiques dans ces pays. Ces données peuvent être demandées lors des procédures de recrutement par le service $\mathrm{RH}$, mais ne sont alors pas communiquées à la ligne hiérarchique en charge du recrutement dans la mesure où elles ne doivent pas rentrer en ligne de compte dans les critères de recrutement. Dans les pays anglo-saxons, la photo du candidat n'a jamais fait partie d'un CV classique. Par contre, les autres éléments figuraient dans le CV, il y a plus de 20 ans alors qu'actuellement, ces données semblent inappropriés pour un CV « moderne », voire politiquement correct ! 


\section{Perception du concept de diversité : une deuxième enquête exploratoire}

\subsection{Contexte, objectif et méthodologie}

Dans le cadre de notre recherche exploratoire, nous avons également souhaité évaluer les concordances et les discordances qu'en France, étudiants d'une part et professionnels de l'autre, pouvaient avoir sur le contenu perçu des différents concepts de la diversité. Nous avons recruté, au premier semestre 2008, 14 étudiants de deuxième cycle d'études en économie, gestion et en droit ainsi que 23 professionnels exerçant en France. Les deux populations sont issues de l'Université de Nice Sophia-Antipolis. Une passation individuelle en face- face a eu lieu pour l'échantillon des étudiants à la bibliothèque de l’Université. Une passation individuelle par e-enquête a eu lieu pour le groupe des professionnels suite à l'exploitation par deux vagues de e-mailing du fichier des 635 anciens étudiants du certificat d'aptitude à l'administration des entreprises. Les questionnaires des deux populations ont été adaptés à partir du questionnaire administré aux étudiants internationaux. L’architecture du questionnaire comportait deux questions ouvertes de demandes de définitions puis les participants étaient interrogés sur leur cursus de formation ou d'information à l'université ou en entreprise sur la RSE et la diversité. Nous leur avons demandé par la suite de noter l'importance de différents concepts de diversité. Les étudiants devaient répondre d'une manière générale pour l'ensemble des entreprises alors que les salariés devaient répondre de manière spécifique pour l'entreprise dans laquelle ils travaillaient lors de l'enquête. Enfin, nous avons conclu pour les deux populations par leur intention d'inclure dans leur CV, leur âge, genre, photographie et situation de famille.

\subsection{Résultats}

Le taux de réponses à l'enquête par Internet auprès des professionnels s'est élevé à 3,6\%. Respectivement 13 et 22 questionnaires sur des effectifs initiaux de 14 étudiants et 23 professionnels ont pu être exploités. Pour les deux échantillons, les femmes ne représentaient que 38 et $36 \%$ du total des effectifs. En ce qui concerne les définitions proposées, nous avons noté que les deux populations limitaient fréquemment leur définition de la RSE à la responsabilité des entreprises vis-à-vis des salariés et de l'environnement, alors que les définitions fournies pour la diversité couvraient de manière plus large le champ de la notion académique de diversité. Nous notons que les étudiants ont bénéficié plus fréquemment d'une formation ou d'une information sur la RSE ( $46 \%$ vs $27 \%$ ) et la diversité (54\% vs 14\%, p $<0,05$ ) au cours de leur cursus universitaire que les professionnels sur leurs lieux de travail. Il n’y a pas de différence significative dans les intentions des deux populations d'insérer sur un prochain CV la mention de l'âge, du genre, de la situation familiale ou de la photographie. C'est d'ailleurs l'insertion de la photographie qui recueille les scores d'intention les plus bas avec seulement 59\% des professionnels et $54 \%$ des étudiants (Tableau 3). De plus dans l'échantillon professionnel, la prévalence de l'intention d'occultation de la photographie du CV est féminine à $75 \%$. 
Tableau 3 : Comparaison des taux de formation à la RSE et à la diversité et des souhaits de composition du CV entre étudiants et professionnels résidants en France.

\begin{tabular}{c|c|c|c|}
\cline { 2 - 4 } & Professionnels (n=22) & Etudiants (n=13) & $\begin{array}{c}\text { Test de } \\
\text { Student } \\
\text { Fischer }\end{array}$ \\
\hline $\begin{array}{c}\text { Bénefice d'au moins une } \\
\text { information ou formation } \\
\text { sur la RSE }\end{array}$ & $27 \%$ & $46 \%$ & $\mathrm{n} . \mathrm{s}$ \\
$\begin{array}{c}\text { Bénéfice d'au moins une } \\
\text { information ou formation } \\
\text { sur la diversité }\end{array}$ & $14 \%$ & $54 \%$ & $\mathrm{p}=0,02$ \\
\hline $\begin{array}{c}\text { Souhait d'insérer l'âge sur } \\
\text { le CV }\end{array}$ & $91 \%$ & $69 \%$ & $\mathrm{~ns}$ \\
$\begin{array}{c}\text { Souhait d'insérer le genre } \\
\text { sur le CV }\end{array}$ & $86 \%$ & $92 \%$ & $\mathrm{~ns}$ \\
$\begin{array}{c}\text { Souhait d'insérer une photo } \\
\text { sur le CV }\end{array}$ & $54 \%$ & $\mathrm{~ns}$ \\
$\begin{array}{c}\text { Souhait d'insérer la } \\
\text { situation famille sur le CV }\end{array}$ & $59 \%$ & $85 \%$ & $\mathrm{~ns}$ \\
\hline
\end{tabular}

En ce qui concerne l'importance accordée aux différentes dimensions de la diversité pour les entreprises en général (selon la vision des étudiants) et pour leur entreprise en particulier (selon la vision des professionnels) nous constatons des écarts significatifs entre les deux échantillons. Les scores d'importance attribués par la population étudiante sont plus élevés que les scores attribués par la population professionnelle. L'importance des critères genre, handicap et race, est significativement supérieure pour les deux populations et dans les deux processus que sont le recrutement et l'évolution de carrière. Pour les professionnels, le score maximal d'importance est obtenu par le critère âge et le score minimum par le critère orientation sexuelle et cela tant pour les procédures de recrutements que pour celles des évolutions de carrières. Alors que pour les étudiants, nous retrouvons un score minimum pour l'orientation sexuelle pour les deux procédures ;c'est l'attention accordée au genre et celle accordée à l'âge, qui atteignent les scores les plus élevés lors respectivement des procédures de recrutements et d'évolutions de carrières. (Tableau 4 et Figure 3) 
Tableau 4: Scores d'importance des composants de la diversité évalués par les étudiants et les professionnels lors des procédures de recrutement et de développement de carrière.

\begin{tabular}{|c|c|c|c|c|}
\hline & & Professionnels $(n=22)$ & Etudiants $(n=13)$ & $\begin{array}{c}\text { Test t de } \\
\text { Student } \\
\text { Fischer }\end{array}$ \\
\hline \multirow{5}{*}{$\begin{array}{l}\text { Importance de } \\
\text { prise en compte } \\
\text { lors des } \\
\text { processus de } \\
\text { recrutement }\end{array}$} & genre * & 3,29 & 5,85 & $p<0,05$ \\
\hline & handicap * & 3,17 & 4,78 & $p<0,05$ \\
\hline & race * & 2,06 & 5,83 & $p<0,05$ \\
\hline & âge & 3,83 & 4,78 & n.s \\
\hline & Orientation sexuelle & 1,84 & 3,38 & n.s \\
\hline \multirow{5}{*}{$\begin{array}{l}\text { Importance de } \\
\text { prise en compte } \\
\text { lors des } \\
\text { processus } \\
\text { d'évolution de } \\
\text { carrière }\end{array}$} & genre ** & 2,80 & 5,25 & $p<0,05$ \\
\hline & handicap ** & 2,43 & 4,43 & $\mathrm{p}<0,05$ \\
\hline & race ** & 1,84 & 5,25 & $p<0,05$ \\
\hline & âge ** & 3,34 & 5,83 & $p<0,05$ \\
\hline & Orientation sexuelle ** & 1,55 & 4,43 & $p<0,05$ \\
\hline
\end{tabular}

Figure 3 : Importance comparée des critères de diversité dans la perception des étudiants et des professionnels lors des procédures de recrutements et d'évolution de carrières

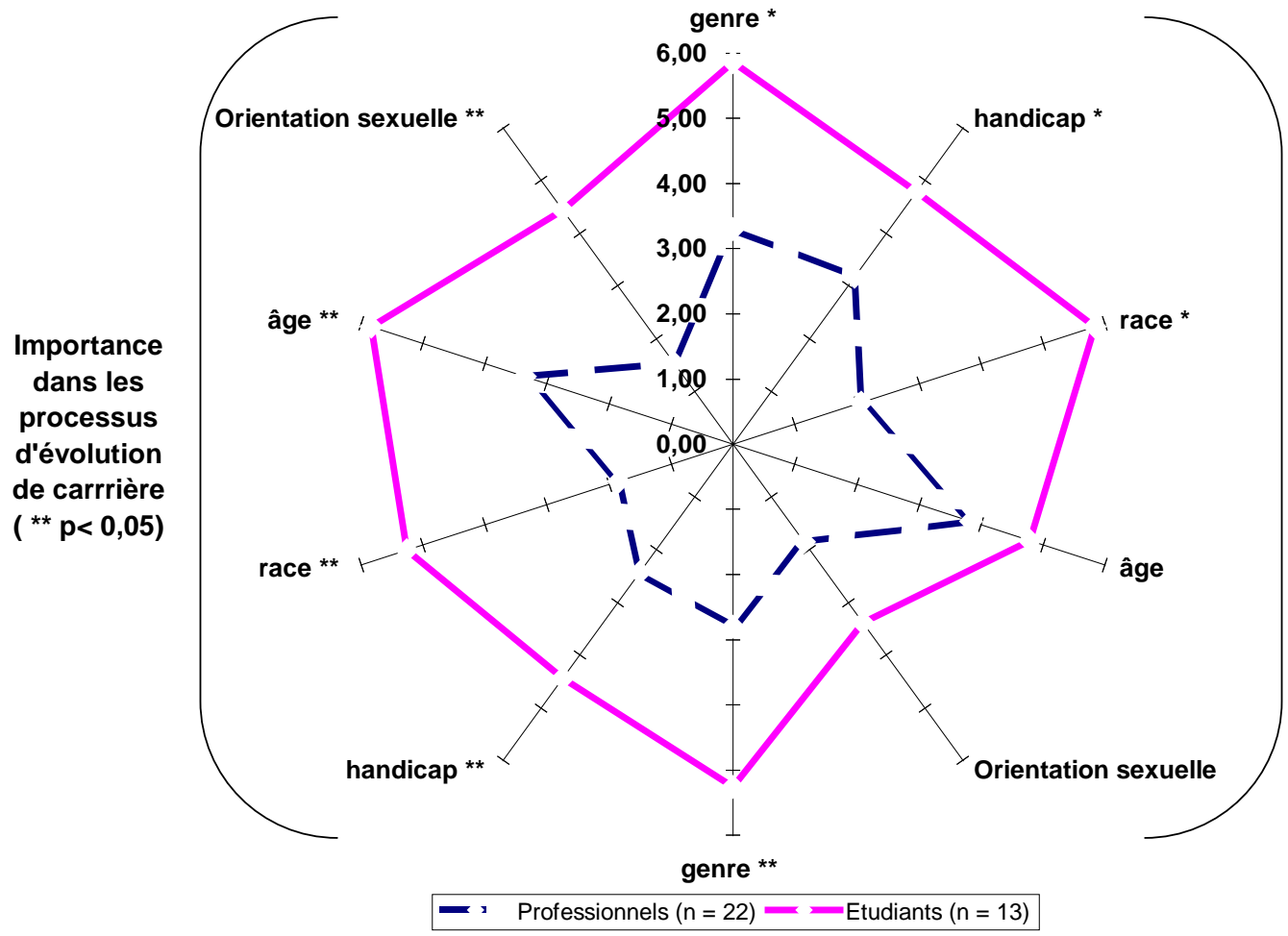

Importance

dans les

processus de recrutement

$\left({ }^{*} p<0,05\right)$ 


\section{Conclusion : les limites, perspectives de recherche et applications}

\section{managériales}

Les limites de notre étude sont principalement celle de l'approche exploratoire avec de faibles effectifs et une hétérogénéité des échantillons qui ne permet pas de comparaison directe entre étudiants en France et à l'étranger et notamment sur la nature et le contenu des cursus enseignés en matière de RSE et de diversité. Les étudiants internationaux sont tous d'un niveau de troisième année universitaire et n'ont pas toujours suivi dans leurs universités respectives, les électifs de dernière année, plus spécialisés incluant éventuellement des enseignements en RSE et/ou en diversité. Par ailleurs, les questionnaires ont été rédigés en anglais, or la plupart des étudiants internationaux, bien qu'anglophones, n'étaient pas de langue maternelle anglaise. Néanmoins les réponses analysées montrent une bonne compréhension du sujet, ce qui permet de penser que ce biais reste limité. Enfin, il est possible que des effets d'ordre dans l'ordonnancement des questions aient pu influencer certaines réponses. Cette première limite impose de revisiter la recherche en normalisant les procédures de recrutement des populations comme celle de passation des questionnaires. Par ailleurs, on ne peut exclure un effet Hawthorne, même si les questions ouvertes en début de questionnaires montrent dans l'ensemble pour les deux populations une connaissance du champ des définitions de la RSE et de la diversité acceptable. De plus, un mode de recueil de données plus qualitatif, sous forme d'interviews individuelles pourrait, lors de travaux ultérieurs, générer des réponses plus argumentées sur la logique des réponses fournies et le potentiel d'évocation suscité par celles-ci.

En ce qui concerne le contingent français, nous constatons que les taux de formation déclarés par les étudiants français sont proches de ceux déclarés par les étudiants internationaux (46\% vs $40 \%$ pour la formation en RSE et $54 \%$ vs $42 \%$ pour la formation à la diversité) d'autant que le cursus universitaire des étudiants français n'étant pas achevé lors de l'enquête (de deuxième cycle) ceux-ci peuvent avoir l'occasion de travailler ultérieurement sur ces sujets. Toutefois, avec seulement $14 \%$ des effectifs des professionnels versus $54 \%$ des effectifs des étudiants français, le taux de formation et d'information à la diversité exprimé par les professionnels semble d'autant plus faible que la formulation de la question était relativement large en mentionnant "dans au moins l'une des entreprises dans lesquelles vous avez travaillé ».. Par ailleurs, la comparaison entre les deux populations françaises sur l'importance accordée aux critères de diversité lors des procédures de recrutement et d'évolution de carrière est signifiante même si son interprétation doit se faire avec prudence. En effet, l'absence d'expérience professionnelle des étudiants ne permet qu'une perception idéalisée des pratiques en entreprise et s'oppose au réalisme de professionnels en situation effective sur leur lieu de travail. En témoigne le verbatim manuscrit ajouté par l'un des participants étudiant concernant l'importance des critères de diversité : " tous ont une importance équivalente car c'est le rejet des discriminations qui est leur point commun ». Une enquête auprès d'une population d'étudiants ayant effectué des stages conséquents en entreprise apporterait des informations probablement différentes. Enfin, même si notre travail est d'abord un état des lieux, il apparaît évident qu'en attendant le renforcement des pratiques de diversité en entreprise par des étudiants spécifiquement formés, une implication plus importante des professionnels passe par un accroissement de la formation continue afin de lutter contre la rémanence des stéréotypes et la réalité des situations de discrimination. Une telle approche permettrait de faire évoluer la diversité en entreprise du stade des pratiques déclaratives au stade de l’intégration dans les flux habituels des activités. 


\section{Bibliographie}

AFP (Agence France Presse) (2007), " Air France, accusée de faire du fichage ethnique », L’Express.fr. http://www.l'express.fr/outils/imprimer/.asp?id=521270.

Barney J. (1991), " Firm Resources and sustained competitive Advantage», Journal of Management, $\mathrm{N}^{\circ} 17$, p.99-120.

Frinck D., Robinson R., Reitel B, Arthur M., Ameter A., Ferris G., Kaplan D., Morisette H., (2003), « Gender Demography and Organization Performance: a two study investigation with convergence », Group and Organization Management, $N^{\circ} 28$.

Ghiulamila J. \& Levet P. (2006), «La mixité, empêtrée dans les stéréotypes. Dossier : des hommes, des femmes, des entreprises », L’Expansion Management Review, N¹21,

Gisbert J. (2007), « Managing Diversity in the work place », EMCC Company Network Seminar. Grant R. (1991), « Resource-Based Theory of competitive advantage: implication for strategy formulation », California Management Review, №33, p.114-135.

Kandolla R. \& Fullerton J. (1994), « Diversity, more than just an empty slogan », Personal Management, Vol.4, $\mathrm{N}^{\circ} 26$, p. 46-50.

Konrad A. \& F.Linnehan, (1995), « Formalized HRM structures: coordinating Equal Employment Opportunity or concealing Organizational Practices? » Academy of Management Journal, Vol.3, No 38, p.387-820.

Liff S. (1997), «Two routes to managing diversity: individual differences or social group characteristics ", Employee relations, Vol.19, N¹.

Litvin D. (1997), " The Discourse of Diversity: From Biology to Management », Organization, $\mathrm{N}^{\circ} 4$, p.187-210.

Millikens F. \& Martins L. (1996), " Searching for common treads: understanding the Multiple Effects of Diversity on Organizational groups ", Academy of Management Review, Vol. 2, No 21, p.402-424.

Picq T. (2005), « La gestion des ressources humaines face au défi de la diversité : relier les performances économiques et sociales », MBA, l'essentiel du management par les meilleurs professeurs, Edition d'Organisation, Paris.

Rijamampianina R. \& Carmichael T. (2005), « General issues in management a pragmatic and holistic approach to managing diversity », Problems and Perspectives in Management, p.109117, January

Semache S. (2006), « La diversité au cœur des équipes : quels enjeux ? », Revue Management et Avenir, $\mathrm{N}^{\circ} 10$, cahier spécial : la diversité, octobre.

Thomas D. et Ely J. (1996), « Making differences matter: a new paradigm for managing diversity », Havard Business Review, Managing Diversity, p.33-66.

Thornburg L. (1994), « Journey toward a more inclusive culture », HR Magazine, Vol.2, N³2, p.79-96.

\section{Webographie}

www.ucas.ac.uk

www.prospects.ac.uk

www.campusfrance.org 